\title{
Dedifferentiated chondrosarcoma with leukocytosis and elevation of serum G-CSF. A case report
}

\author{
Akio Sakamoto*1, Hidetaka Yamamoto², Kazuhiro Tanaka1, \\ Shuichi Matsuda1, Katsumi Harimaya1, Yoshinao Oda², \\ Masazumi Tsuneyoshi ${ }^{2}$ and Yukihide Iwamoto ${ }^{1}$
}

\author{
Address: ${ }^{1}$ Department of Orthopaedic Surgery, Graduate School of Medical Sciences, Kyushu University, Fukuoka, 812-8582, Japan and \\ ${ }^{2}$ Department of Anatomic Pathology, Graduate School of Medical Sciences, Kyushu University, Fukuoka, 812-8582, Japan \\ Email: Akio Sakamoto* - akio@med.kyushu-u.ac.jp; Hidetaka Yamamoto - hidetaka@surgpath.med.kyushu-u.ac.jp; \\ Kazuhiro Tanaka - tanaka@ortho.med.kyushu-u.ac.jp; Shuichi Matsuda - mazda@ortho.med.kyushu-u.ac.jp; \\ Katsumi Harimaya - harimaya@ortho.med.kyushu-u.ac.jp; Yoshinao Oda - oda@surgpath.med.kyushu-u.ac.jp; \\ Masazumi Tsuneyoshi - masazumi@surgpath.med.kyushu-u.ac.jp; Yukihide Iwamoto - yiwamoto@ortho.med.kyushu-u.ac.jp \\ * Corresponding author
}

Published: 04 July 2006

World Journal of Surgical Oncology 2006, 4:37 doi:10.1 186/1477-78/9-4-37

This article is available from: http://www.wjso.com/content/4/1/37

(c) 2006 Sakamoto et al; licensee BioMed Central Ltd.

This is an Open Access article distributed under the terms of the Creative Commons Attribution License (http://creativecommons.org/licenses/by/2.0), which permits unrestricted use, distribution, and reproduction in any medium, provided the original work is properly cited.
Received: 19 February 2006

Accepted: 04 July 2006

\begin{abstract}
Background: G-CSF is known to function as a hematopoietic growth factor and it is known to be responsible for leukocytosis. G-CSF-producing tumors associated with leukocytosis include various types of malignancies.

Case presentation: We report the case of a 72-year-old man with dedifferentiated chondrosarcoma characterized by dedifferentiated components of malignant fibrous histiocytomaor osteosarcoma-like features in addition to conventional chondrosarcoma, arising from his pelvic bone. After hemipelvectomy, when local recurrence and metastasis were identified, leukocytosis appeared and an elevated level of serum granulocyte-colony-stimulating factor (G-CSF) was also recognized. The patient died of multiple organ failure 2 months after surgery. Autopsy specimens showed that the histological specimens of the recurrence and metastasis were dedifferentiated components, without any conventional chondrosarcoma components. G-CSF was expressed only in the dedifferentiated components, not in the chondrosarcoma components, immunohistochemically.
\end{abstract}

Conclusion: This is the first report of chondrosarcoma, or any other primary bone tumor, with leukocytosis, probably stimulated by tumor-produced G-CSF from the dedifferentiated components.

\section{Background}

Granulocyte-colony stimulating factor (G-CSF) enhances differentiation along the neutrophil lineage, and accelerates maturation of metamyelocytes into mature neutrophils. G-CSF also prolongs the survival of neutrophils and their precursors, including stem cells[1]. Consequently, G-CSF is known to function as a hematopoietic growth factor and it is known to be responsible for leukocytosis. Normally, the serum G-CSF level is very low [2]. Production of granulocyte colony-stimulating factor (G- 
CSF) by tumor cells was first identified in lung carcinoma in 1977 [3]. G-CSF-producing tumors associated with leukocytosis include various types of malignancies, including lung caner [4,5], colon cancer [4], stomach cancer [4], thyroid caner [6], cervical cancer [7], malignant fibrous histiocytoma of soft tissue [8].

Dedifferentiated chondrosarcoma accounts for approximately $10 \%$ of all chondrosarcomas [9]. Dedifferentiated chondrosarcoma shows both a rapid growth rate and metastatic spread, and it has a significantly worse survival rate compared with conventional chondrosarcoma $[10,11]$. Dedifferentiated chondrosarcoma shows both a rapid growth rate and metastatic spread, and it has a significantly worse survival rate compared with conventional chondrosarcoma. The overall 5-year survival rate in conventional chondrosarcoma patients varies between $48 \%$ $60 \%$, on the other hand, dedifferentiated chondrosarcoma is highly lethal, with less than a $10 \%$ survival rate after 1 year, with practically no long-term survivors [9]. Dedifferentiated chondrosarcoma is characterized by the coexistence of conventional chondrosarcoma and dedifferentiated components. The dedifferentiated components can features of malignant fibrous histiocytoma $(\mathrm{MFH})$, osteosarcoma, angiosarcoma, fibrosarcoma, rhabdomyosarcoma, leiomyosarcoma and giant cell tumor [11]. We report a case of dedifferentiated chondrosarcoma with leukocytosis, probably due to stimulation by tumor-produced G-CSF. This chondrosarcoma with leukocytosis and an elevated serum G-CSF level is the first such report not only of chondrosarcoma, but also of any such primary bone tumor with leukocytosis.

\section{Case presentation}

A 72-year-old man, who had been suffering from right hip pain for 7 years when walking, was referred to our institute. Roentogenography of the right pelvic bone showed an expansion of the cortical contour and a soft-tissue mass with punctate calcification (figure 1). Magnetic resonance imaging (MRI) showed a lobulated lesion expanding over the entire right pelvic bone with low-intensity on T1weighted imaging and iso- to high-intensity on T2weighted imaging, with the tumor protuberant to the pelvic cavity (figure 1). Right hemipelvectomy was carried out. Histological sections showed a conventional chondrosarcoma having atypical chondrocytes with hyaline cartilage matrix or myxoid matrix (figure 2). In a small section, dedifferentiated components were also identified, and these dedifferentiated components consisted of atypical spindle cells arranged in short fascicles or a storiform pattern showing MFH-like features, plus some pleomorphic cells with lace-like osteoid formation showing osteosarcoma-like features (figure 3). Therefore, the final diagnosis was dedifferentiated chondrosarcoma because of the coexistence of conventional chondrosarcoma and dedifferentiated components.

Preoperative laboratory data were not remarkable. After surgery, the laboratory data also showed leukocytosis predominantly in the neutrophils with an elevated level of Creactive protein (figure 4 ). The serum level of G-CSF was also elevated (330 pg/ml [normal, $<8 \mathrm{pg} / \mathrm{ml}]$ ). Magnetic resonance imaging (MRI) and computed tomography (CT) revealed evidence of local recurrence and metastatic lesions in the lungs. Flow-cytometry indicated no evidence of leukemia and serological studies showed no evidence of specific infections, such as candidiasis or tuberculosis. Despite the administration of several antibiotics, the leukocytosis did not disappear. Two months after the surgery, the patient died of multiple organ failure. An autopsy was carried out, and in addition to the lung metastasis, metastasis was also found in the liver, thyroid, diaphragm, adrenal gland, digestive tract and skin. The histology of the recurrent and metastatic lesions was not conventional chondrosarcoma but only dedifferentiated components. Immunoexpression of G-CSF (antiG-CSF [Ab1], Calbiochem, San Diego CA, USA) was seen in the dedifferentiated components, but not in the conventional chondrosarcoma components (figure 5).

\section{Discussion}

In the current report, we present a case of dedifferentiated chondrosarcoma with leukocytosis and an increased serum G-CSF level. In a previous report, serum G-CSF was not found to be increased in bone tumors, including osteosarcoma, chondrosarcoma, Ewing/PNET and giant cell tumor, compared to the control [12]. Therefore, an elevation of serum G-CSF seemed to be a specific finding in the current case. Leukocytosis in the current case was probably stimulated by tumor-produced G-CSF. G-CSF-producing tumors associated with leukocytosis are a well-known phenomenon. Besides tumor cells themselves, neoplastic necrosis produces multiple cytokines, including G-CSF, and causes leukecytosis [15]. However, there is less necrotic area in this dedifferentiated chondrosarcoma. Moreover, the degree of leukocytosis associated with surgery, such as reactive local inflammation and healing wound, is mild, which is different from leukocytosis caused by tumor-produced G-CSF. Taken together, the leukocytosis seemed to be strongly associated with an elevated serum G-CSF of neoplastic origin. Secondary nonepithelial tumors with leukocytosis have been reported, among which there was a case of undifferentiated sarcoma following a fracture [13] and a case of angiosarcoma following burns [14]. Among the primary bone and soft-tissue tumors, only 5 cases of soft-tissue tumor have been reported, among which there was one case of MFH [8] and 4 cases of liposarcoma [15-18]. However, to our knowl- 

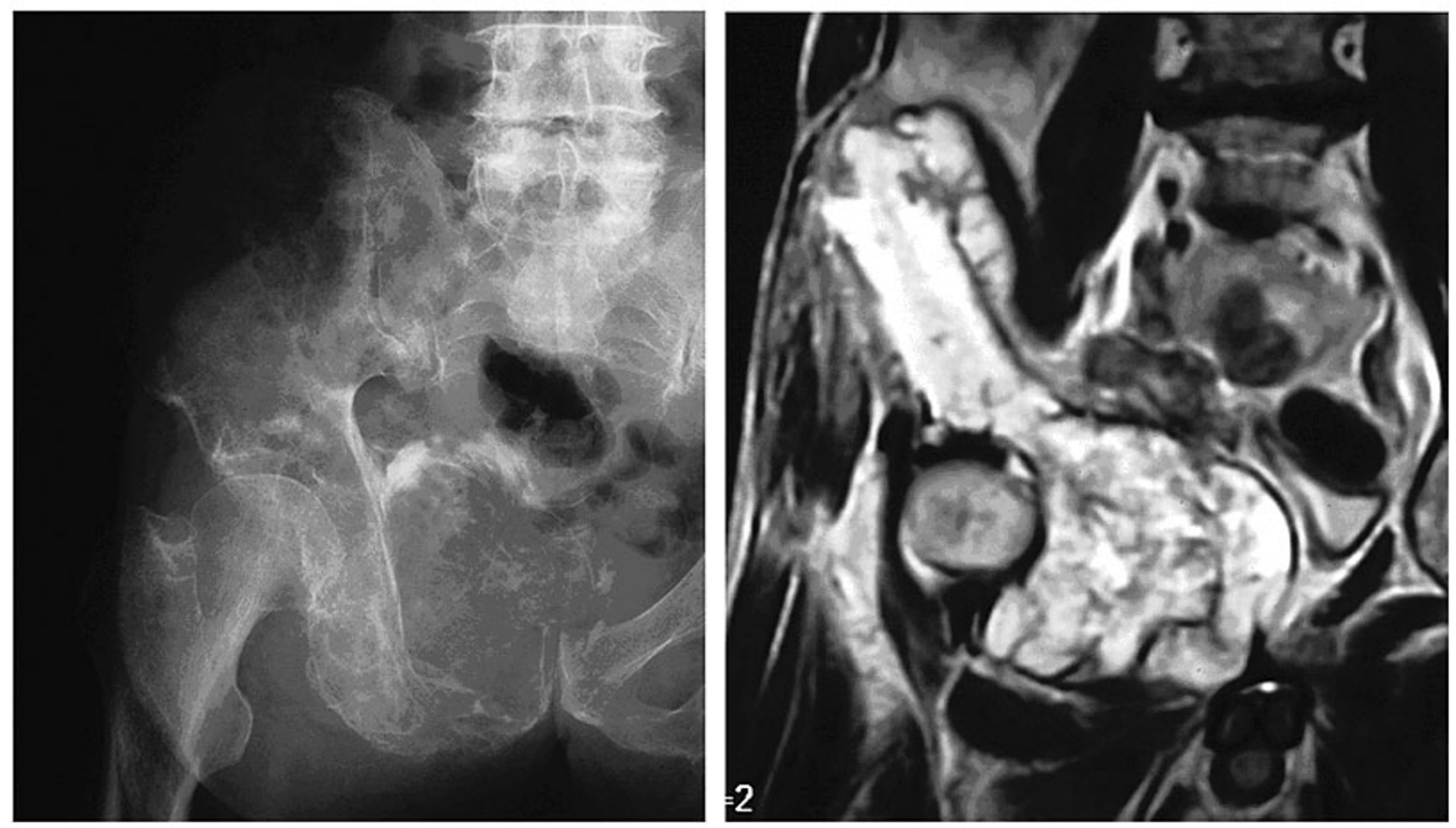

\section{Figure I}

Roentgenographs show an expansive lesion with punctate calcification in the right pelvic bone (left). MRI shows a lobulated lesion, protuberant to the pelvic cavity, in the pelvic bone with high-intensity on T2-weighted image (right).

edge, no bone tumor with leukocytosis plus an elevated serum G-CSF level has been reported.

In the current case, leukocytosis appeared after the surgery, when recurrence and metastasis also appeared. The histology of the recurrent and metastatic lesions was not conventional chondrosarcoma but only dedifferentiated components. Therefore, it would seem that the G-CSF production could have been associated with the dedifferentiated components. Supporting this idea, G-CSF expression was found only in the dedifferentiated components, and not in the conventional chondrosarcoma components, immunohistochemically. As described, the histology of G-CSF-producing soft-tissue tumors was MFH and liposarcoma [8,15-18]. The subtype of these reported liposarcoma cases was pleomorphic type $[15,16]$ and dedifferentiated type $[17,18]$, in 2 cases each. G-CSF production in bone and soft-tissue tumors may be related with undifferentiated sarcoma, such as MFH or sarcomas of pleomorphic type or dedifferentiated type.

The current case of dedifferentiated chondrosarcoma had a rather long history of 7 years without leukocytosis prior to the initial surgery. The resected specimens had conven- tional chondrosarcoma components in most part, and dedifferentiated components only in a small part. It is not clear why there was no leukocytosis before the surgery, even though there were dedifferentiated components. One theory is that the volume of dedifferentiated components with G-CSF production was too small to cause leukocytosis. Another theory is that there was no production at the beginning, and dedifferentiated components acquired the function of G-CSF production during the course of tumor development. When leukocytosis appeared, the tumor had already recurred and metastasis had developed, and the patient died 2 months after the surgery. G-CSF has been shown to induce tumor cell proliferation [19-21]. In addition, in a previous report, tumor-related leukocytosis is linked with poor prognosis in patients with lung carcinoma. Patients with lung carcinoma with leukocytosis had a poor outcome compared with the other patients without leukocytosis [5]. In the same line, G-CSF may have contributed to the aggressive behavior of dedifferentiated chondrosarcoma in the current case.

It is uncertain whether dedifferentiated chondrosarcoma consists primarily of two cell types or whether dedifferen- 

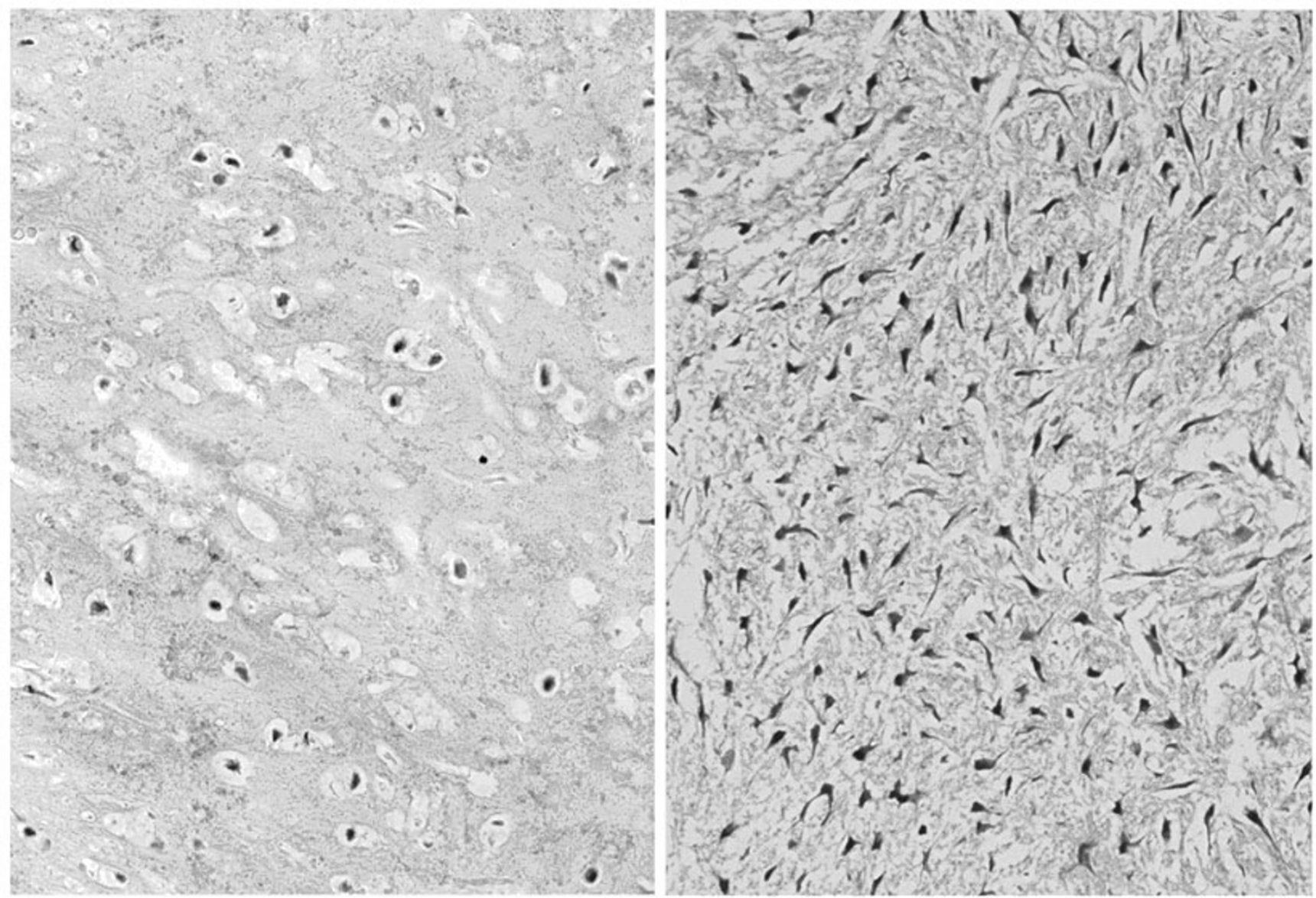

\section{Figure 2}

Dedifferentiated chondrosarcoma; well-differentiated components show conventional chondrosarcoma composed of atypical chondrocytes with hyaline cartilage matrix (left) and with myxoid matrix (right) (Hematoxylin and Eosin original magnification $\times(80)$.

tiation develops secondarily. Molecular genetic study of one case has shown a substantial number of genetic alterations, as well as severe aneuploidy and loss of heterozygosity in both the components of dedifferentiated chondrosarcoma [22]. LOH (loss of heterozygosity) in Rb predominantly occurs in the dedifferentiated components of dedifferentiated chondrosarcomas [23]. Gene alterations in $p 53, R b$ and FHIT are reported to be responsible for the "switch" to a high-grade anaplastic sarcoma (dedifferentiated components) from monoclonal origin of demonstrated dedifferentiated chondrosaroma [24]. It is also possible that the function of G-CSF production was acquired as a result of genetic alteration during the course of tumor development in the current case.

\section{Conclusion}

In this report, we present a case of dedifferentiated chondrosarcoma with leukocytosis. The leukocytosis seemed to be associated with an elevated serum G-CSF level, which may be related to dedifferentiated components in the current dedifferentiated chondrosarcoma case.

\section{Competing interests}

The author(s) declare that they have no competing interests.

\section{Authors' contributions}

AS drafted the manuscript and carried out the immunohistochemical studies. HY and YO carried out the immunohistochemical studies. KT, SM and KH participated in the design of the study and performed. MT and YI conceived of the study, and participated in its design and coordination and helped to draft the manuscript. All authors read and approved the final manuscript.

\section{Acknowledgements}

The patient's families were informed that data from the case would be submitted for publication, and gave their consent. 

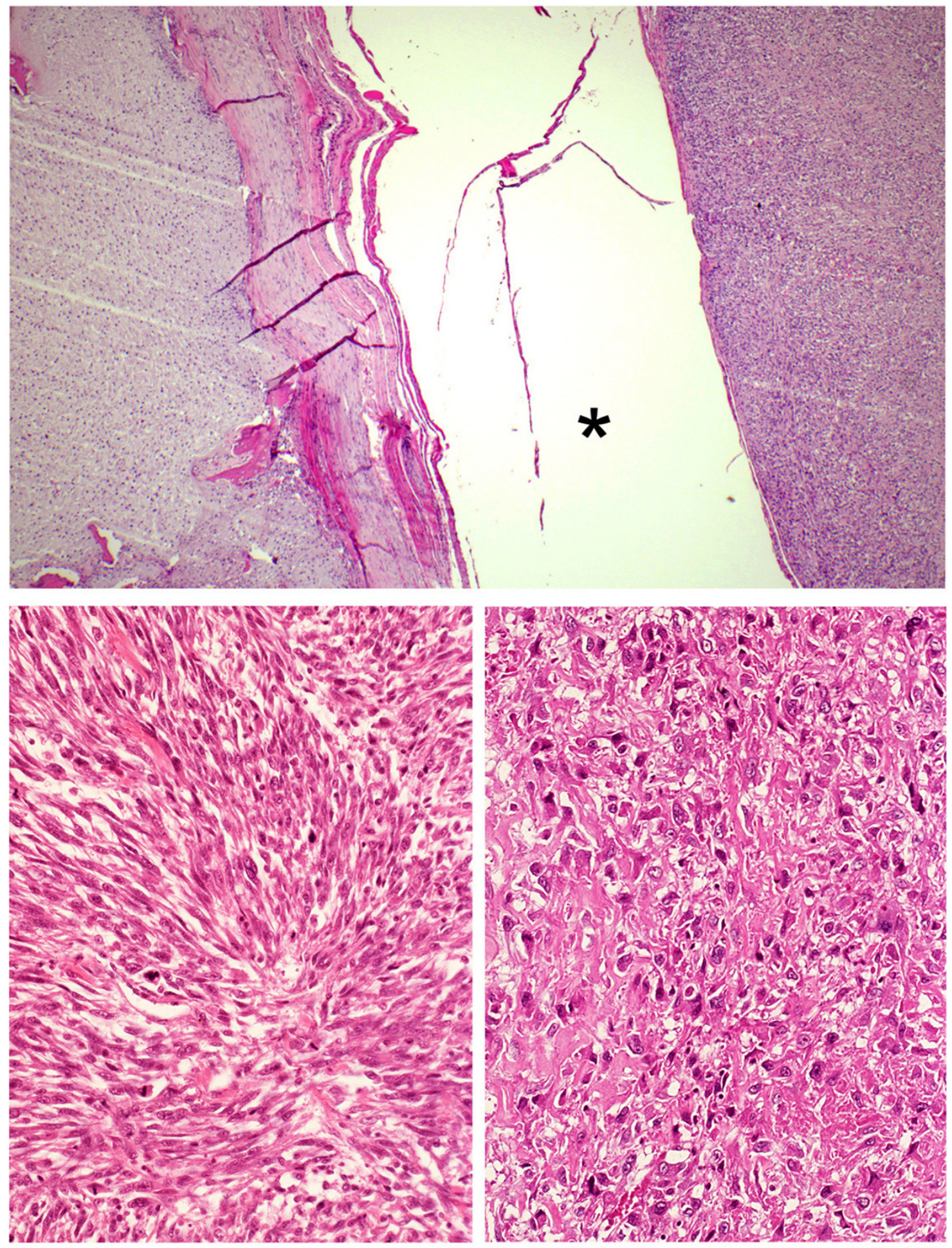

\section{Figure 3}

Dedifferentiated chondrosarcoma is characterized by the coexistence of conventional chondrosarcoma (top; left side) and dedifferentiated components (top; right side). Dedifferentiated components are composed of atypical spindle cells in a storiform pattern, resembling MFH (bottom; left), or osteosarcoma with lace-like osteoid formation (bottom; right). Asterisk $\left(^{*}\right)$ shows artificial space. (Hematoxylin and Eosin original magnification top; $\times 50$, bottom; $\times 180$ ). 


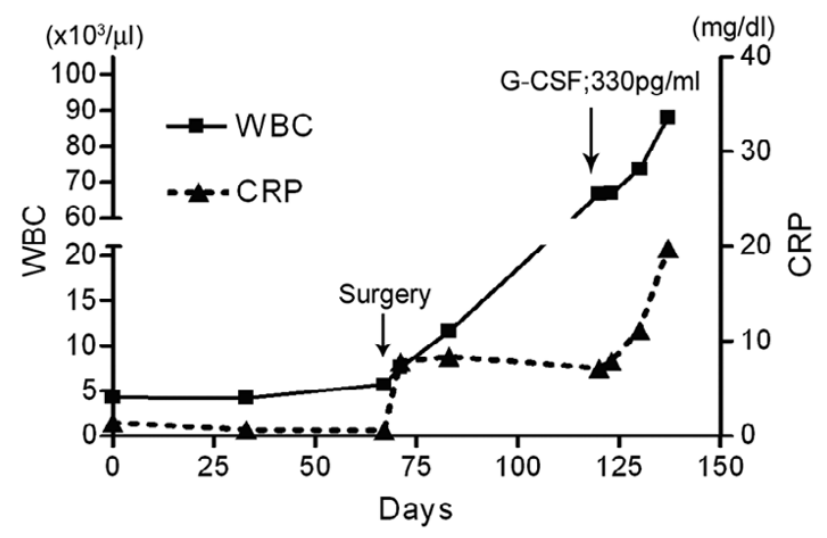

Figure 4

Leukocytosis appeared after the surgery. Elevated serum GCSF level is shown (arrow). C-reactive protein is also increased.
The English used in this manuscript was revised by Miss K. Miller (Royal English Language Centre, Fukuoka, Japan).

\section{References}

I. Roberts AW: G-CSF: a key regulator of neutrophil production, but that's not all! Growth factors 2005, 23(I):33-4I.

2. Cebon J, Layton JE, Maher D, Morstyn G: Endogenous haemopoietic growth factors in neutropenia and infection. $\mathrm{Br} J$ Haematol 1994, 86(2):265-274.

3. Asano S, Urabe A, Okabe T, Sato N, Kondo Y: Demonstration of granulopoietic factor(s) in the plasma of nude mice transplanted with a human lung cancer and in the tumor tissue. Blood 1977, 49(5):845-852.

4. Kojima K, Nakashima F, Boku A, Muroishi Y, Nakanishi I, Oda Y: Clinicopathological study of involvement of granulocyte colony stimulating factor and granulocyte-macrophage colony stimulating factor in non-lymphohematopoietic malignant tumors accompanied by leukocytosis. Histol Histopathol 2002, I7(4): $1005-1016$.

5. Kasuga I, Makino S, Kiyokawa $\mathrm{H}$, Katoh $\mathrm{H}$, Ebihara $\mathrm{Y}$, Ohyashiki K: Tumor-related leukocytosis is linked with poor prognosis in patients with lung carcinoma. Cancer 200I, 92(9):2399-2405.

6. Sato T, Omura M, Saito J, Hirasawa A, Kakuta Y, Wakabayashi $Y$, Nishikawa T: Neutrophilia associated with anaplastic carcinoma of the thyroid: production of macrophage colony-stimulating factor (M-CSF) and interleukin-6. Thyroid 2000, IO(I2): $1113-1118$.

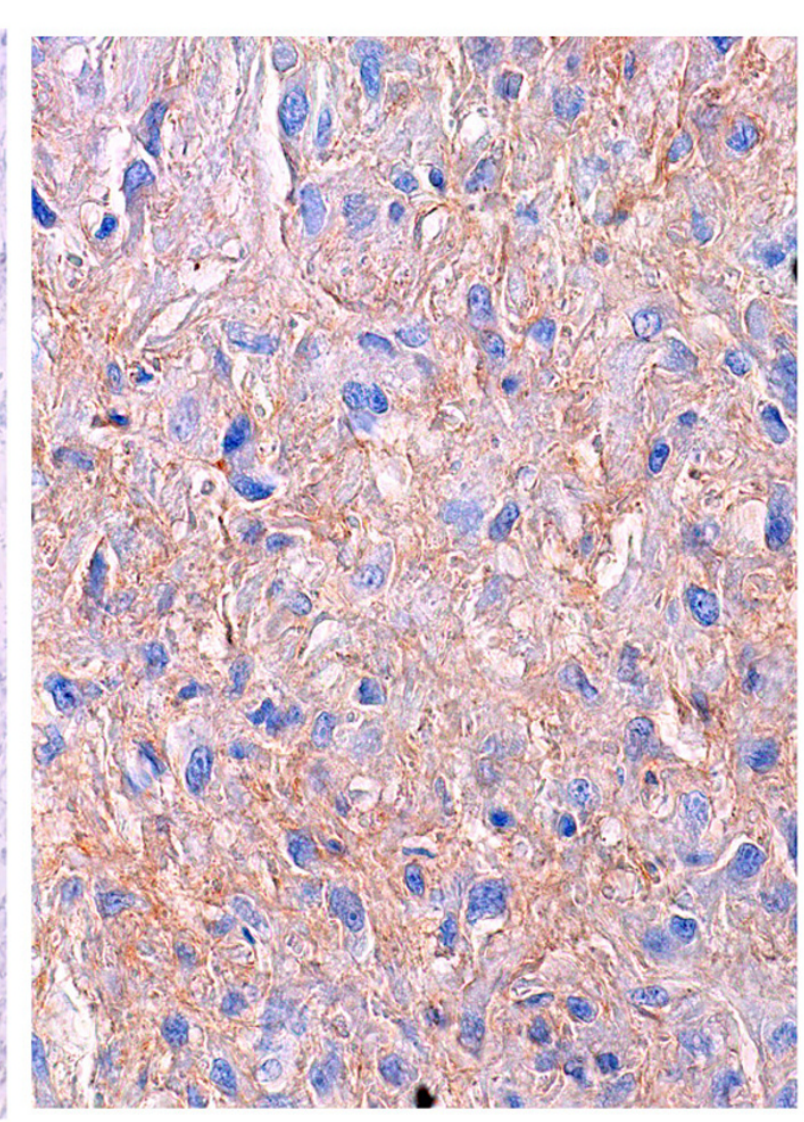

\section{Figure 5}

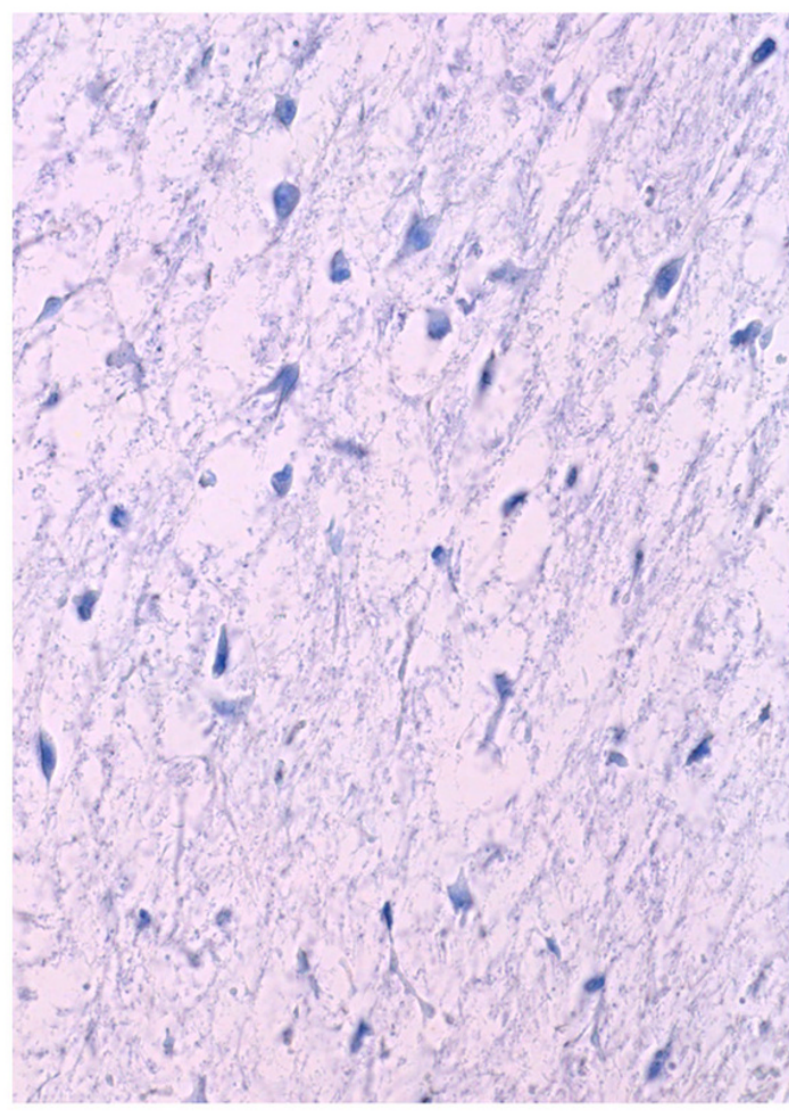

Immunohistochemistry of G-CSF is absent in the conventional chondrosarcoma components (left), while is seen in the dedifferentiated components (right) (Immunohistochemistry original magnification $\times 250$ ). 
7. Kyo S, Kanaya T, Takakura M, Inoue M: A case of cervical cancer with aggressive tumor growth: possible autocrine growth stimulation by G-CSF and II-6. Gynecol Oncol 2000, 78(3 Pt I):383-387.

8. Mayumi E, Okuno $T$, Ogawa $T$, Kurata $K$, Ishioka $H$, Hamamoto $H$, Ishiki K, Maga T, Okamoto T, Yoshida T, Ogura Y: Malignant fibrous histiocytoma of soft tissue producing granulocyte colony-stimulating factor. Intern Med 200I, 40(6):536-540.

9. Dorfman HD, Czerniak B: Bone Tumors. St Louis , Mosby; 1998:353-440.

10. Sakamoto A, Oda Y, Adachi T, Oshiro Y, Tamiya S, Tanaka K, Matsuda $\mathrm{S}$, Iwamoto $\mathrm{Y}$, Tsuneyoshi $\mathrm{M}$ : $\mathbf{H}$-ras oncogene mutation in dedifferentiated chondrosarcoma: polymerase chain reaction-restriction fragment length polymorphism analysis. Mod Pathol 200I, I4(4):343-349.

II. Bruns J, Fiedler W, Werner M, Delling G: Dedifferentiated chondrosarcoma--a fatal disease. J Cancer Res Clin Oncol 2005, |3 |(6):333-339.

12. Rutkowski P, Kaminska J, Kowalska M, Ruka W, Steffen J: Cytokine and cytokine receptor serum levels in adult bone sarcoma patients: Correlations with local tumor extent and prognosis. J Surg Oncol 2003, 84(3): I5I-I59.

13. Marui T, Yamamoto T, Akisue T, Hitora T, Yoshiya S, Kurosaka M: Granulocyte colony-stimulating factor-producing undifferentiated sarcoma occurring in previously fractured femur. A case report and review of the literature. Arch Pathol Lab Med 2003, I 27(4): el 86-9.

14. Nara T, Hayakawa A, Ikeuchi A, Katoh N, Kishimoto S: Granulocyte colony-stimulating factor-producing cutaneous angiosarcoma with leukaemoid reaction arising on a burn scar. $\mathrm{Br} J$ Dermatol 2003, I 49(6): | 273-1275.

15. Matsumoto M, Yazawa Y, Kanzaki M: An autopsy case of liposarcoma with granulocytic leukemoid reaction. Acta Pathol Jpn 1976, 26(3):399-408.

16. Nakamura A, Tanaka S, Takayama H, Sakamoto $M$, Ishii $H$, Kusano $M$, Onizuka $Y$, Ota $S$, Mitamura K: A mesenteric liposarcoma with production of granulocyte colony-stimulating factor. Intern Med 1998, 37(10):884-890.

17. Hisaoka M, Tsuji S, Hashimoto H, Aoki T, Uriu K: Dedifferentiated liposarcoma with an inflammatory malignant fibrous histiocytoma-like component presenting a leukemoid reaction. Pathol Int 1997, 47(9):642-646.

18. Nasser SM, Choudry UH, Nielsen GP, Ott MJ: A leukemoid reaction in a patient with a dedifferentiated liposarcoma. Surgery 200I, I 29(6):765-767.

19. Rennick D, Jackson J, Yang G, Wideman J, Lee F, Hudak S: Interleukin-6 interacts with interleukin-4 and other hematopoietic growth factors to selectively enhance the growth of megakaryocytic, erythroid, myeloid, and multipotential progenitor cells. Blood 1989, 73(7):1828-1835.

20. Tachibana M, Miyakawa A, Uchida A, Murai M, Eguchi K, Nakamura K Kubo A, Hata Jl: Granulocyte colony-stimulating factor receptor expression on human transitional cell carcinoma of the bladder. Br J Cancer I997, 75( ( 0): I 489- I 496.

21. Thacker JD, Dedhar S, Hogge DE: The effect of GM-CSF and G. CSF on the growth of human osteosarcoma cells in vitro and in vivo. Int J Cancer 1994, 56(2):236-243.

22. Bovee JV, Cleton-Jansen AM, Rosenberg C, Taminiau AH, Cornelisse C], Hogendoorn PC: Molecular genetic characterization of both components of a dedifferentiated chondrosarcoma, with implications for its histogenesis. J Pathol 1999, 189(4):454-462.

23. Ropke M, Boltze C, Meyer B, Neumann HW, Roessner A, SchneiderStock R: Rb-loss is associated with high malignancy in chondrosarcoma. Oncol Rep 2006, I 5(I):89-95.

24. Ropke M, Boltze C, Neumann HW, Roessner A, Schneider-Stock R: Genetic and epigenetic alterations in tumor progression in a dedifferentiated chondrosarcoma. Pathol Res Pract 2003, 199(6):437-444

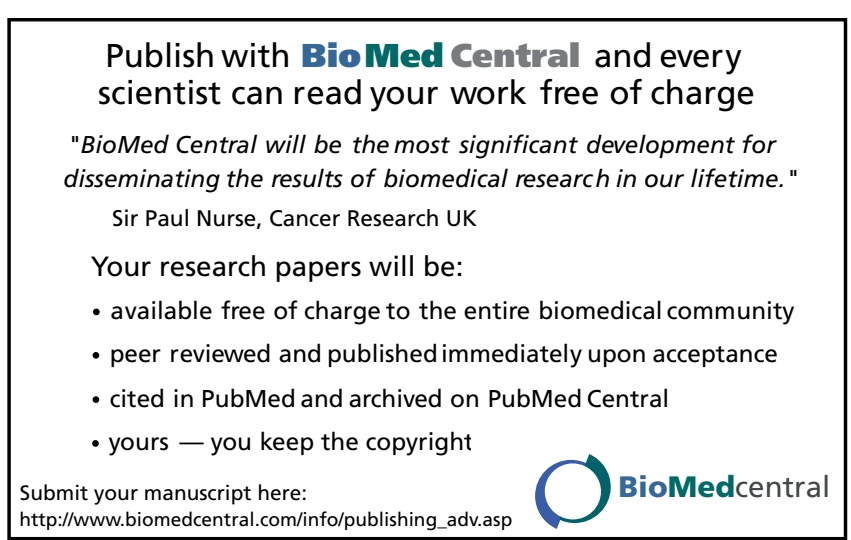

